\title{
Analysis of Entropy Generation in Diffusion HTAC Processes
}

\author{
Deodat Makhanlall and Peixue Jiang, Member, IACSIT
}

\begin{abstract}
Thermodynamic irreversibility significantly limits fuel energy conversion into useful work. Hence, the evaluation of thermodynamic irreversibility is an important aspect in the development of combustion techniques for better fuel utilization. This evaluation is performed for the HTAC (high-temperature air combustion) process through entropy generation analysis. The primary irreversible processes of HTAC are determined and the influence of key operating parameters such as the combustion air temperature, and oxygen-dilution are studied.
\end{abstract}

Index Terms - Combustion, entropy generation, HTAC mode, thermodynamics.

\section{INTRODUCTION}

HTAC (high-temperature air combustion) is an emerging combustion technique that offers the important benefit of fuel savings. Burning of less fuel means reduced greenhouse gas emissions. Combustion in the HTAC mode is also distinctive by its strong flame stability and homogeneity [1]. In addition, HTAC processes have significantly increased radiation heat transfer rates [1]. Furnaces operating in HTAC mode may however exhibit an important drawback: the increase of the combustion air temperature promotes NO-formation [2]-[4].

Recently, many studies have been conducted to investigate NO-formation in HTAC processes. These studies show that an oxygen-diluted environment is required for suppressing NO-formation [3]-[6]. In addition to NO-related issues, there are other aspects that still need be studied to provide adequate insight into HTAC processes. For instance, thermodynamic analyses of the HTAC mode are still lacking. Hence, this paper assesses the thermodynamic irreversibilities of HTAC. Such irreversibilities cause entropy generation which limits fuel energy conversion into useful work [7].

\section{MATHEMATICAL Modeling}

\section{A. Computational Methodology}

The study is carried out with the FLUENT ${ }^{\circledR} 6.3$ CFD code. Fig. 1 shows the problem setup, which is 2-D axis-symmetric. Computational details are specified in Table I. The HTAC process is modeled using the two-step reaction mechanism with CO intermediates [8]. The RNG k-e turbulence model is used, while turbulence-chemistry interaction is solved using the finite-rate/eddy dissipation model. This model, computes

Manuscript received March 3, 2014; revised May 12, 2014. This work is supported financially by Tsinghua University.

The authors are with the Tsinghua University, the Department of Thermal Engineering, Beijing, $100084 \quad$ PR China (e-mail: deodat@mail.tsinghua.edu.cn, jiangpx@mail.tsinghua.edu.cn). both the Arrhenius and Eddy-dissipation reaction rates, and takes the minimum of these two rates as the net reaction rate [9]. The models for thermal radiation are:

$$
\begin{aligned}
& \mathbf{s} \cdot \nabla I_{\lambda}(\mathbf{r}, \mathbf{s})=-\left(\kappa_{a \lambda}+\kappa_{s \lambda}\right) I_{\lambda}(\mathbf{r}, \mathbf{s})+\kappa_{a \lambda} I_{\lambda}(\mathbf{r}, \mathbf{s}) \\
& +\frac{\kappa_{s \lambda}}{4 \pi} \int_{4 \pi} I_{\lambda}\left(\mathbf{r}, \mathbf{s}^{\prime}\right) \Phi\left(\mathbf{s}^{\prime}, \mathbf{s}\right) \mathrm{d} \Omega . \\
& I_{\lambda}\left(\mathbf{r}_{\mathbf{w}}, \mathbf{s}\right)=\varepsilon_{w} I_{b, \lambda}\left(\mathbf{r}_{\mathbf{w}}\right)+\frac{1-\varepsilon_{w}}{\pi} \int_{n \cdot s^{\prime}<0} I_{\lambda}\left(\mathbf{r}_{\mathbf{w}}, \mathbf{s}^{\prime}\right)\left|\mathbf{n}_{\mathbf{w}} \cdot \mathbf{s}^{\prime}\right| \mathrm{d} \Omega
\end{aligned}
$$

with

$$
\Phi\left(\mathbf{s}^{\prime}, \mathbf{s}\right)=1+\mathbf{s}^{\prime} \cdot \mathbf{s}
$$

Here, $\kappa_{a \lambda}$ and $\kappa_{s \lambda}$ are the spectral absorption and spectral scattering coefficients, respectively. They are determined by a weighted sum of gray gas model. $I_{\lambda}$ is the spectral radiative intensity, $I_{b \lambda}$ is the spectral blackbody intensity, $\Omega$ is the solid angle, $\Phi$ is the scattering phase function, $\varepsilon_{w}$ is the wall emissivity, $\mathbf{s}$ is a unit position vector, $\mathbf{r}$ is a unit direction vector, and $\mathbf{n}_{w}$ is an outward unit normal vector.

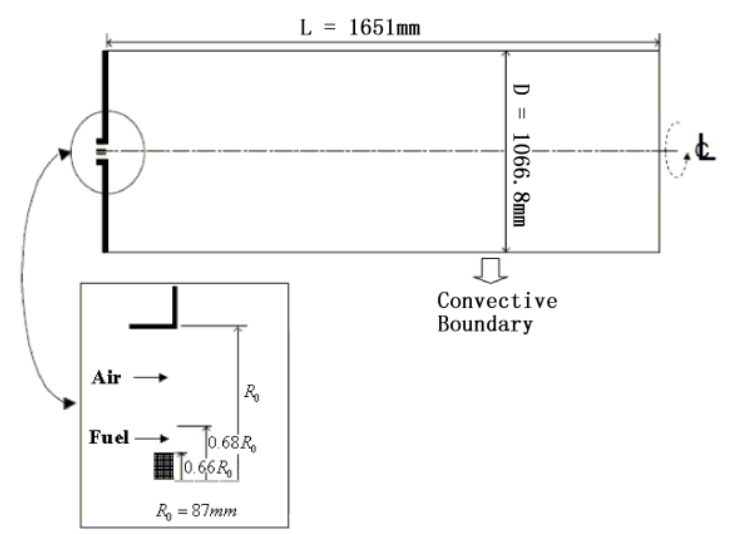

Fig. 1. Problem schematic.

TABLE I: COMPUTATIONAL DETAILS

\begin{tabular}{lll}
\hline \hline Parameter & SDC & HTAC \\
\hline Inlet Air Temperature $(\mathrm{K})$ & 300 & $400-1200$ \\
Inlet oxygen volume fraction $(\%)$ & 21 & $10-20$ \\
Fuel-air ratio & 0.022 & 0.022 \\
Absorption coefficient $\left(\mathrm{m}^{-1}\right)$ & WSGG & WSGG \\
Scattering coefficient $\left(\mathrm{m}^{-1}\right)$ & 0.01 & 0.01 \\
Wall emissivity & 0.7 & $0.5-1$ \\
Wall heat transfer coefficient $\left(\mathrm{Wm}^{-2} \mathrm{~K}^{-1}\right)$ & 10 & 10 \\
\hline \hline
\end{tabular}




\section{B. Entropy Generation}

Entropy generation is a post-processing quantity. Once the governing equations are solved, the local volumetric rate of entropy generation is computed as following [10]:

$$
\dot{S}_{g e n}^{\prime \prime \prime}=\dot{S}_{g e n, f}^{\prime \prime \prime}+\dot{S}_{g e n, c h}^{\prime \prime \prime}+\dot{S}_{g e n, m}^{\prime \prime \prime}+\dot{S}_{g e n, c}^{\prime \prime \prime}+\dot{S}_{g e n, r}^{\prime \prime \prime}
$$

where

$$
\begin{gathered}
\dot{S}_{g e n, f}^{\prime \prime \prime}=\frac{\mu_{e f f} \Psi}{T} \\
\dot{S}_{g e n, c h}^{\prime \prime \prime}=\frac{\sum_{i} \dot{\omega}(-\nabla H) \bar{M}_{w}}{T} \\
\dot{S}_{g e n, m}^{\prime \prime \prime}=\frac{-\sum_{i} J_{i, e f f} \nabla \chi_{i}}{T} \\
\dot{S}_{g e n, r}^{\prime \prime \prime a e s}=\int_{0}^{\infty} \int_{4 \pi}\left[-\left(\kappa_{a \lambda}+\kappa_{s \lambda}\right) \frac{I_{\lambda}(\mathbf{r}, \mathbf{s})}{T_{\lambda}(\mathbf{r}, \mathbf{s})}+\kappa_{s} \frac{I_{b \lambda}(\mathbf{r})}{T_{\lambda}(\mathbf{r}, \mathbf{s})}\right. \\
\left.+\frac{\kappa_{s \lambda}}{4 \pi} \int_{4 \pi} \frac{I_{\lambda}\left(\mathbf{r}, \mathbf{s}^{\prime}\right)}{T_{\lambda}(\mathbf{r}, \mathbf{s})} \Phi\left(\mathbf{s}, \mathbf{s}^{\prime}\right) \mathrm{d} \Omega^{\prime}\right] \mathrm{d} \Omega \mathrm{d} \lambda \\
\left.+\int_{0}^{\infty} \int_{4 \pi} \frac{\kappa_{a \lambda}}{T}(\nabla T)^{2}(\mathbf{r}, \mathbf{s})-I_{b \lambda}(\mathbf{r})\right) \mathrm{d} \Omega \mathrm{d} \lambda
\end{gathered}
$$

with

$$
T_{\lambda}(\mathbf{r}, \mathbf{s})=\frac{h c_{0}}{\lambda k_{b}} \frac{1}{\ln \left[2 h c_{0}^{2} \lambda^{-5} / I_{\lambda}(\mathbf{r}, \mathbf{s})+1\right]}
$$

are the local volumetric entropy generation rates associated with viscous dissipation, chemical reactions, mass transfer, heat conduction and convection, and radiation transfer in the medium, respectively. $\mu_{\text {eff }}, k_{\text {eff }}$, and $J_{i, \text { eff }}$ are the effective viscosity, effective thermal conductivity, and effective mass diffusion flux, respectively. $\Psi$ is the viscous dissipation function, $\dot{\omega}$ is reaction rate, $H$ is enthalpy, $\bar{M}_{w}$ is mean molecular weight, $\chi$ is chemical potential, $h$ is Planck's constant, $k_{b}$ is Boltzmann constant, and $c_{0}$ is speed of light.

Entropy generation due to wall radiation transfer processes need also be considered when computing the overall entropy generation rate [11]. The local radiation entropy generation rate per unit surface area is defined as:

$$
\dot{S}_{g e n, r}^{\prime \prime W}=\int_{0}^{\infty} \int_{4 \pi}\left[\frac{I_{\lambda}\left(\mathbf{r}_{w}, \mathbf{s}\right)}{T\left(\mathbf{r}_{w}\right)}-L_{\lambda}\left(\mathbf{r}_{w}, \mathbf{s}\right)\right]\left(\mathbf{n}_{w} \cdot \mathbf{s}\right) \mathrm{d} \Omega \mathrm{d} \lambda
$$

where

$$
\begin{aligned}
& L_{\lambda}\left(\mathbf{r}_{w}, \mathbf{s}\right)=\frac{2 k_{b} c_{0}}{\lambda^{4}}\left\{\ln \left(\frac{\varepsilon_{w}}{\exp \left(h c_{0} / k_{b} \lambda T\right)-1}+1\right) \times\right. \\
& \left(\frac{\varepsilon_{w}}{\exp \left(h c_{0} / k_{b} \lambda T\right)-1}+1\right)-\ln \left(\frac{\varepsilon_{w}}{\exp \left(h c_{0} / k_{b} \lambda T\right)-1}\right) \times \\
& \left.\left(\frac{\varepsilon_{w}}{\exp \left(h c_{0} / k_{b} \lambda T\right)-1}\right)\right\}
\end{aligned}
$$

The total entropy generation rate is computed by integrating (5)-(9) over volume and (11) over surface area

$$
\dot{S}_{G, t o t}=\dot{S}_{G, f}+\dot{S}_{G, c h}+\dot{S}_{G, m}+\dot{S}_{G, c}+\dot{S}_{G, r}^{a e s}+\dot{S}_{G, r}^{W}
$$

With

$$
\begin{aligned}
& \dot{S}_{G, f}=\int_{V} \dot{S}_{g e n, f}^{\prime \prime \prime} \mathrm{d} V \\
& \dot{S}_{G, c h}=\int_{V} \dot{S}_{g e n, c h}^{\prime \prime \prime} \mathrm{d} V \\
& \dot{S}_{G, m}=\int_{V} \dot{S}_{g e n, m}^{\prime \prime \prime} \mathrm{d} V \\
& \dot{S}_{G, c}=\int_{V} \dot{S}_{g e n, c}^{\prime \prime \prime} \mathrm{d} V \\
& \dot{S}_{G, r}^{a e s}=\int_{V} \dot{S}_{g e n, r}^{\prime \prime \prime a e s} \mathrm{~d} V \\
& \dot{S}_{G, r}^{W}=\int_{A} \dot{S}_{g e n, r}^{\prime \prime \prime} \mathrm{dA}
\end{aligned}
$$

\section{RESUlts AND DisCUSSION}

Volumetric entropy generation rates of the HTAC mode are shown in Fig. 2. Comparison is made with the standard diffusion combustion (SDC) mode. Volumetric entropy generation rates due to viscous dissipation, the chemical reactions, mass transfer, and heat conduction and convection are much lower in the HTAC diffusion flame. These reduced volumetric entropy generation rates are due to the strong homogeneity of the HTAC mode [1]. However, radiation entropy generation rates are increased in the HTAC process.

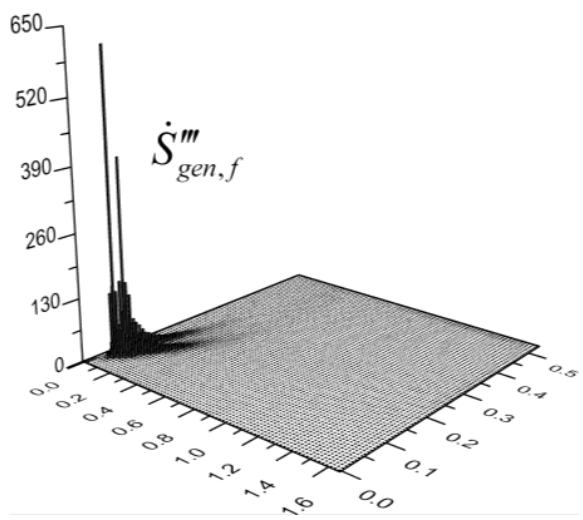



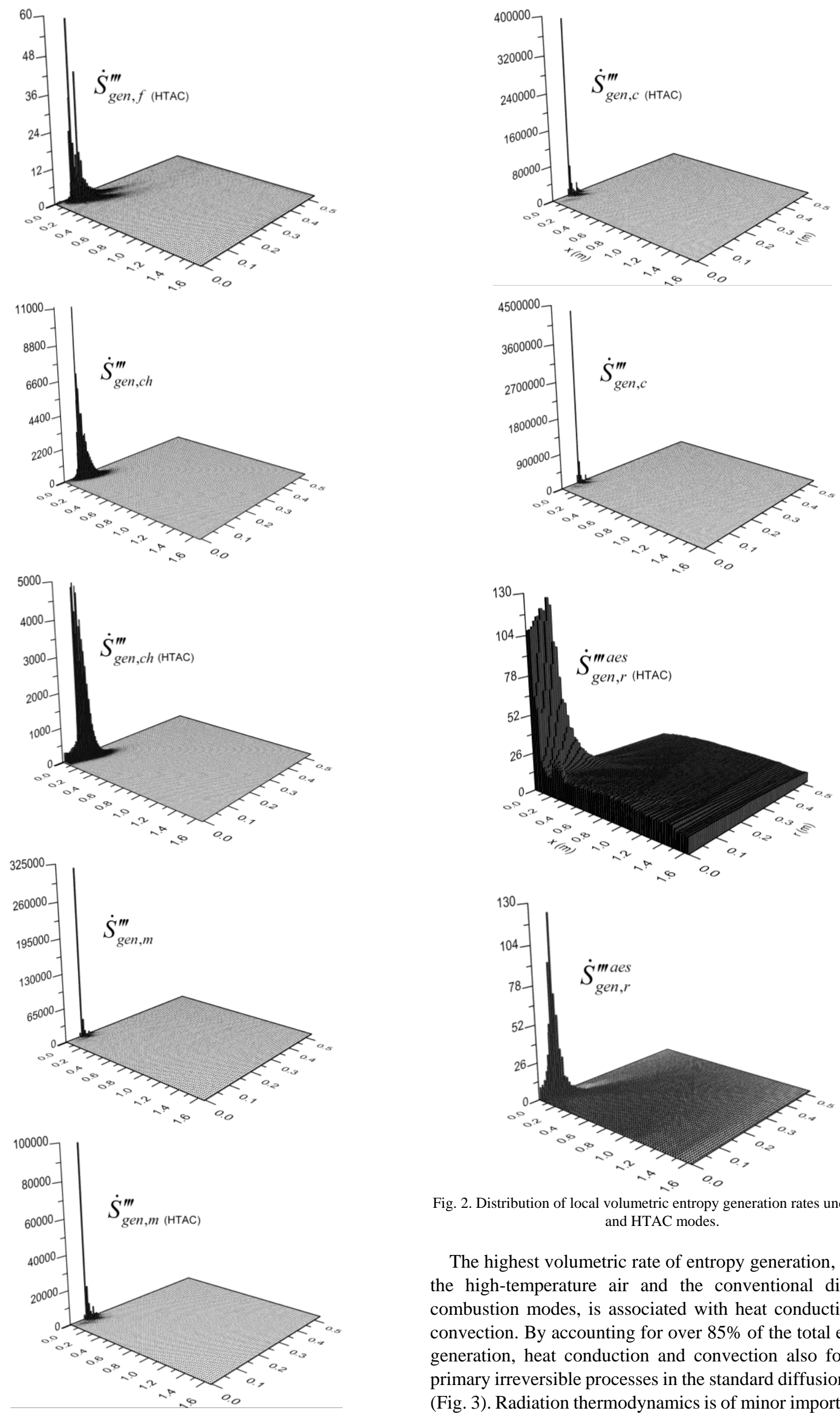

Fig. 2. Distribution of local volumetric entropy generation rates under SDC and HTAC modes.

The highest volumetric rate of entropy generation, in both the high-temperature air and the conventional diffusion combustion modes, is associated with heat conduction and convection. By accounting for over $85 \%$ of the total entropy generation, heat conduction and convection also form the primary irreversible processes in the standard diffusion flame (Fig. 3). Radiation thermodynamics is of minor importance in 
the conventional combustion process. However, the effects of thermal radiation cannot be neglected in the HTAC mode.

Thermal radiation accounts for more than $80 \%$ of the overall entropy generation in the HTAC process (Fig. 3). This shift from heat conduction and convection to thermal radiation as the main irreversible process is due largely to the pre-heated air temperature. It is well known that radiative heat transfer, and its associated thermodynamic effects, becomes more significant at elevated temperatures [10].

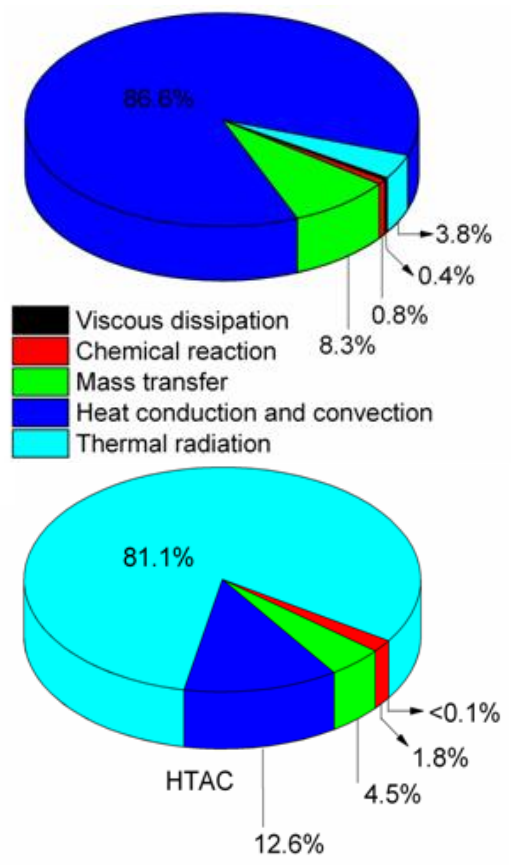

Fig. 3. Impact of various processes on entropy generation in HTAC mode.

Fig. 4 depicts the relationship between air temperature and entropy generation rate. With increasing air temperature, the overall radiation entropy generation rate increases strongly, while overall entropy generation rate due to heat conduction and convection reduces. The increase of radiation entropy generation rate is primarily due to wall radiation transfer processes. Radiation entropy generation in the medium becomes relatively less important with increasing air temperature. Fig. 4 also shows that in HTAC processes the overall entropy generation rate first reduces and then increases with air temperature. Overall entropy generation rate is minimum at $T=550 \mathrm{~K}$.

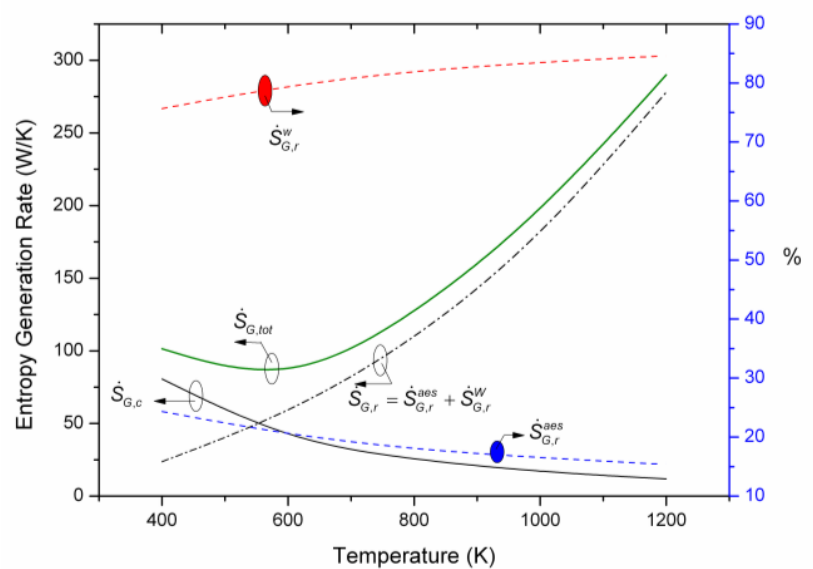

Fig. 4. Effect of combustion air temperature on entropy generation in HTAC mode (oxygen volume fraction $15 \%$, wall emissivity 0.7 ).
Oxygen-dilution is one of the primary features of the HTAC mode because of its effect on NO-formation [1]. The effect of oxygen-dilution on entropy generation in the HTAC mode is depicted in Fig. 5. The overall radiation entropy generation rate increases, principally due to wall radiation processes, while entropy generation associated with heat conduction and convection reduces as the oxygen volume fraction in air becomes lower. The overall thermodynamic effect of diluted air in the HTAC mode involves a small increase of the overall entropy generation rate.
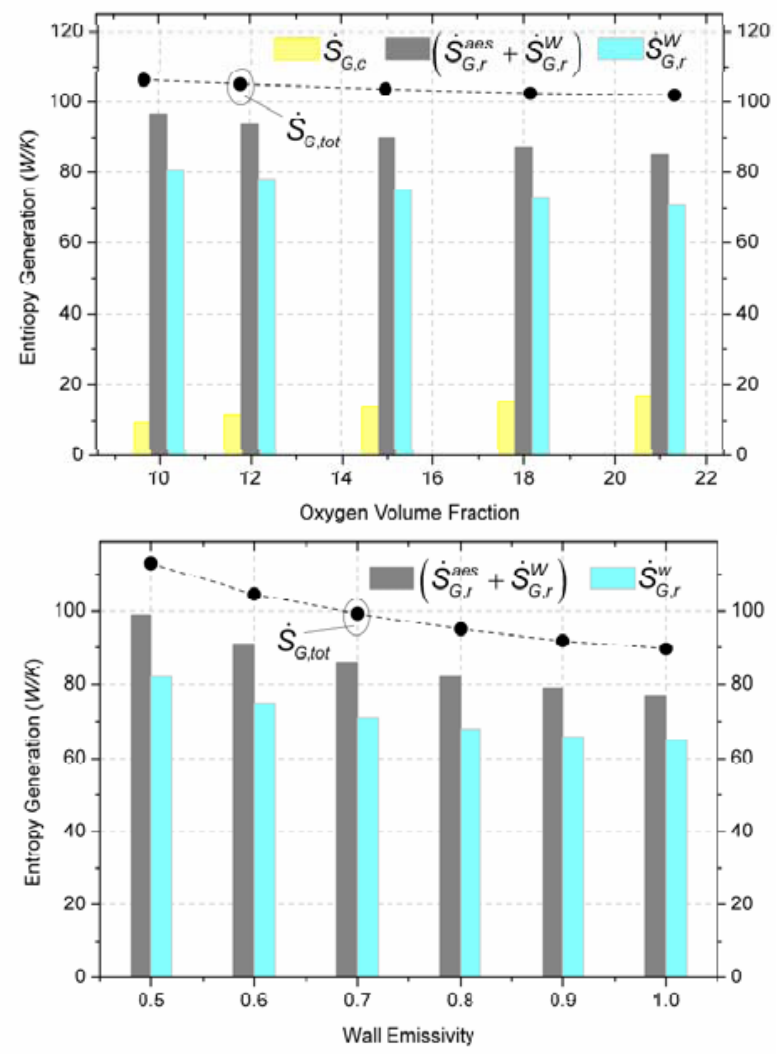

Fig. 5. Effect of oxygen volume fraction of inlet combustion air and wall emissivity on entropy generation in HTAC mode (air temperature is $1000 \mathrm{~K}$ ).

For the HTAC mode, thermal radiation transfer processes at solid walls is the main mechanism of entropy generation. The wall emissivity is an important parameter that controls wall radiation heat transfer. Wall emissivity also affects radiation entropy generation at solid walls. Fig. 5 shows that the overall radiation entropy generation rate reduces with increasing wall emissivity. Overall entropy generation in the HTAC process also reduces with increasing wall emissivity. Thus, high wall emissivity values should be selected in order to reduce thermodynamic irreversibilities in the HTAC mode.

\section{CONCLUSION}

Compared to standard diffusion combustion, entropy generation associated with viscous dissipation, mass transfer, chemical reactions and heat conduction and convection has significantly reduced rate in diffusion HTAC processes. Radiation entropy generation rates are however strongly increased in HTAC processes. Thermal radiation is the main cause of entropy generation in HTAC flames. Radiation entropy generation rates increase strongly with the preheated 
air temperature. This increase is due to wall radiation processes. The wall radiation entropy generation can be reduced by increasing wall emissivity. Overall entropy generation rate in HTAC can also be minimized by selecting an appropriate air temperature. The oxygen volume fraction in the combustion air has only a small affect on the overall entropy generation rate in HTAC processes.

\section{REFERENCES}

[1] H. Tsuji, A. K. Gupta, T. Hasegawa, M. Katsuki, K. Kishimoto, and M. Morita, High Temperature Air Combustion: From Energy Conservation to Pollution Reduction, Boca Raton: CRC, 2003, ch. 2 , pp. 29-51.

[2] K. W. Lee and D. H. Choi, "Numerical study on high-temperature diluted air combustion for the turbulent jet flame in crossflow using an unsteady flamelet model," International Journal of Heat and Mass Transfer, vol. 52, no. 25-26, pp. 5740-5750, Dec. 2009.

[3] M. Nishimura, T. Suzuki, R. Nakanashi, and R. Kitamura, "Low-NOx combustion under high preheated air temperature condition in an industrial furnace," Energy Convers. Mgmt., vol. 38, no. 10-13, pp. 1353-1363, March 1997.

[4] S. Lille, W. Blasiak, and M. Jewartowski, "Experimental study of the fuel jet combustion in high temperature and low oxygen content exhaust gases," Energy, vol. 30, no. 2-4, pp. 373-384, March 2005.

[5] G. Choi and M. Katsuki, "Advanced low NOx combustion using highly preheated air," Energy Convers. Mgmt., vol. 42, no. 5, pp. 639-652, March 2001.

[6] R. Weber, S. Orsino, N. Lallemant, and A. Verlaan, "Combustion of natural gas with high-temperature air and large quantities of flue gas," in Proc. Combust. Inst., vol. 28, no. 1, pp. 1315-1321, 2000.
[7] S. K. Som and A. Datta, "Thermodynamic irreversibilities and exergy balance in combustion processes," Prog. Energy Combust. Sci., vol. 34, pp. 351-376, June 2008 .

[8] C. K. Westbrook and F. L. Dryer, "Simplified reaction mechanisms for oxidation of hydrocarbon fuels in flames," Combust. Sci. Technol., vol. 27, no. 1-2, pp. 31-43, July 1981.

[9] FLUENT 6.3 User's Guide, Lebanon, 2006, ch. 15, pp. 15(1)-15(95).

[10] D. Makhanlall, J. L. Munda, and P. Jiang, "Radiation energy devaluation in diffusion combusting flows of natural gas," Energy, vol. 61, no. 1-2, pp. 657-663, July 2013.

[11] L. H. Liu and S. X. Chu, "Verification of numerical simulation method for entropy generation of radiation heat transfer in semitransparent medium," Energy, vol. 61, no. 1-2, pp. 657-663, July 2013.

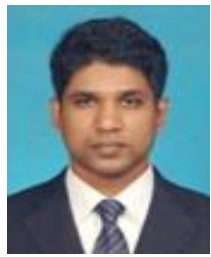

Deodat Makhanlall is a senior member of IACSIT. He obtained his $\mathrm{PhD}$ in engineering thermophysics from Harbin Institute of Technology (HIT), Harbin, PR China, in June 2010. He is a research associate at Tsinghua University, Dept. of Thermal Engineering, Beijing, PR China. His area of research is thermodynamic analysis of fluid flow and heat transfer processes.

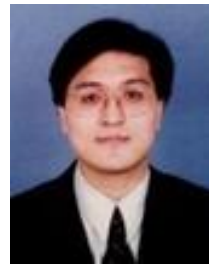

Jiang Peixue obtained his PhD from Moscow Power Engineering Institute, Moscow, Russia in February 1991. He is an executive director of General Scientific R\&D Office at Tsinghua University and also the director of Institute of Engineering Thermophysics. He has carried out extensive research on nano-scale thermal transport, transpiration cooling, and thermal treatment of sludge. 